\title{
Anomalous magnetic moments from asymptotic safety
}

\author{
Gudrun Hiller $\odot,{ }^{1}$ Clara Hormigos-Feliu $\odot,{ }^{1}$ Daniel F. Litim $\odot,{ }^{2}$ and Tom Steudtner ${ }^{2}$ \\ ${ }^{1}$ Fakultät Physik, TU Dortmund, Otto-Hahn-Straße 4, D-44221 Dortmund, Germany \\ ${ }^{2}$ Department of Physics and Astronomy, University of Sussex, Brighton BNI 9QH, United Kingdom
}

(Received 26 November 2019; accepted 24 August 2020; published 15 October 2020)

\begin{abstract}
The measurements of the muon and electron anomalous magnetic moments hint at physics beyond the standard model. We show why and how models inspired by asymptotic safety can explain deviations from standard model predictions naturally. Our setup features an enlarged scalar sector and Yukawa couplings between leptons and new vectorlike fermions. Using the complete two-loop running of couplings, we observe a well-behaved high-energy limit of models including a stabilization of the Higgs. We find that a manifest breaking of lepton universality beyond standard model Yukawas is not necessary to explain the muon and electron anomalies. We further predict the tau anomalous magnetic moment and new particles in the TeV energy range, whose signatures at colliders are indicated. With small $C P$ phases, the electron EDM can be as large as the present bound.
\end{abstract}

DOI: 10.1103/PhysRevD.102.071901

\section{INTRODUCTION}

Measurements of the electron and muon anomalous magnetic moments exhibit intriguing discrepancies from standard model (SM) predictions [1-3]. Adding uncertainties in quadrature, the deviations

$$
\begin{aligned}
& \Delta a_{\mu} \equiv a_{\mu}^{\exp }-a_{\mu}^{\mathrm{SM}}=268(63)(43) \times 10^{-11} \\
& \Delta a_{e} \equiv a_{e}^{\exp }-a_{e}^{\mathrm{SM}}=-88(28)(23) \times 10^{-14}
\end{aligned}
$$

amount to $3.5 \sigma(2.4 \sigma)$ for the muon (electron). Recent theory predictions for $a_{\mu}$ find up to $4.1 \sigma$ [4,5]. There are two stunning features in the data. First, the deviations $\Delta a_{\mu}$ and $\Delta a_{e}$ have opposite sign. Second, their ratio $\Delta a_{e} / \Delta a_{\mu}=-(3.3 \pm 1.6) \times 10^{-4}$ is an order of magnitude smaller than the lepton mass ratio $m_{e} / m_{\mu}$ and an order of magnitude larger than the square of the mass ratio $\left(m_{e} / m_{\mu}\right)^{2}$. Theory explanations of the data (1), with either new light scalars [6-9], supersymmetry [10-12], bottom-up models $[13,14]$, or others $[15,16]$, manifestly break lepton flavor universality.

In recent years, asymptotic safety has been put forward as a new idea for model building $[17,18]$. It is based on the discovery [19] that particle theories may very well remain fundamental and predictive in the absence of asymptotic freedom due to interacting high-energy fixed points [20-22]. For weakly coupled theories, general theorems for

Published by the American Physical Society under the terms of the Creative Commons Attribution 4.0 International license. Further distribution of this work must maintain attribution to the author(s) and the published article's title, journal citation, and DOI. Funded by SCOAP . asymptotic safety are available $[23,24]$ with templates covering simple [19,25,26], semisimple [27], and supersymmetric gauge theories [28]. Yukawa interactions and new scalar fields play a prominent role because they slow down the growth of asymptotically nonfree gauge couplings, which can enable interacting fixed points [23], including in extensions of the standard model $[17,18,29,30]$.

In this paper, we show that asymptotically safe extensions of the SM may offer a natural explanation for the data (1). The primary reason for this is that Yukawa interactions, which help to generate interacting fixed points, can also contribute to lepton anomalous magnetic moments. We demonstrate this idea in two concrete models by introducing Yukawa couplings between ordinary leptons and new vectorlike fermions, and by adding new scalar fields which admit either a flavorful or flavor-universal ground state. Unlike in all previous works [6-16], we find that the data (1) can be accommodated without any explicit breaking of lepton universality. The stability of SM extensions all the way up to the Planck scale is exemplified using the renormalization group (RG) running of couplings for a wide range of BSM parameters.

\section{NEW VECTORLIKE FERMIONS AND SCALAR MATTER}

In the spirit of Ref. [19], we are interested in SM extensions involving $N_{F}$ flavors of vectorlike color-singlet fermions $\psi_{i}$ and $N_{F}^{2}$ complex scalar singlets $S_{i j}$. In their simplest form, the new fermions couple to SM matter only via gauge interactions $[17,18]$. The new ingredients in this paper are Yukawa couplings between SM and BSM matter. To make contact with SM flavor, we set $N_{F}=3$. We then consider singlet or doublet models where the new fermions 
are either $S U(2)$ singlets with hypercharge $Y=-1$, or $S U(2)$ doublets with $Y=-\frac{1}{2}$. In our conventions, electric charge $Q$ and weak isospin $T_{3}$ relate as $Q=T_{3}+Y$. Within these choices, and denoting the SM lepton singlets, doublets, and Higgs as $E, L$, and $H$, respectively, we find three possible Yukawa couplings $\kappa, \kappa^{\prime}$, and $y$ with

$$
\begin{aligned}
\mathcal{L}_{\mathrm{Y}}^{\text {singlet }} & =-\kappa \bar{L} H \psi_{R}-\kappa^{\prime} \bar{E} S^{\dagger} \psi_{L}-y \bar{\psi}_{L} S \psi_{R}+\text { H.c. }, \\
\mathcal{L}_{\mathrm{Y}}^{\text {doublet }} & =-\kappa \bar{E} H^{\dagger} \psi_{L}-\kappa^{\prime} \bar{L} S \psi_{R}-y \bar{\psi}_{L} S \psi_{R}+\text { H.c. },
\end{aligned}
$$

and flavor traces are understood to simplify the subsequent RG analysis. Effects of the Yukawa coupling $y$ have been studied in Refs. [17,18,29]. The scalar potential of either model reads

$$
\begin{aligned}
V= & \lambda\left(H^{\dagger} H\right)^{2}+\delta H^{\dagger} H \operatorname{Tr}\left[S^{\dagger} S\right] \\
& +u \operatorname{Tr}\left[S^{\dagger} S S^{\dagger} S\right]+v\left(\operatorname{Tr}\left[S^{\dagger} S\right]\right)^{2},
\end{aligned}
$$

where $u, v, \lambda$, and $\delta$ are quartic and portal couplings. We further introduce mass terms for the scalars and vectorlike fermions. The potential (3) admits vacuum configurations $V^{+}$and $V^{-}$characterized by

$$
\begin{aligned}
& V^{+}:\left\{\begin{array}{l}
\lambda>0, \quad u>0, \quad u+3 v>0, \\
\delta>-2 \sqrt{\lambda(u / 3+v)},
\end{array}\right. \\
& V^{-}:\left\{\begin{array}{l}
\lambda>0, \quad u<0, \quad u+v>0, \\
\delta>-2 \sqrt{\lambda(u+v)}
\end{array}\right.
\end{aligned}
$$

Either of these allows for electroweak symmetry breaking. Moreover, in $V^{+}$, and for suitable mass parameters, the diagonal components of $S$ each acquire the same vacuum expectation value $\left\langle S_{\ell \ell}\right\rangle \neq 0$, and the ground state is flavor universal. In $V^{-}$, a finite vacuum expectation value $\left\langle S_{\ell \ell}\right\rangle \neq 0$ arises only for one flavor direction, giving rise to a flavorful vacuum.

\section{EXPLAINING ANOMALOUS MAGNETIC MOMENTS}

We are now in a position to explain the data (1) in SM extensions with Eqs. (2) and (3). The relevant leading loop effects due to the couplings $\kappa, \kappa^{\prime}$, and $\delta$ are shown in Fig. 1, also using $S=\langle S\rangle+s$. Any lepton flavor $\ell=e, \mu, \tau$ receives a contribution from BSM scalar-fermion loops with chiral flip on the lepton line induced by the coupling $\kappa^{\prime}$ [see Fig. 1(a)]. It scales quadratically with the lepton mass,

$$
\Delta a_{\ell}=\frac{N_{F} \kappa^{2}}{96 \pi^{2}} \frac{m_{\ell}^{2}}{M_{F}^{2}} f_{1}\left(\frac{M_{S}^{2}}{M_{F}^{2}}\right),
$$

and represents a minimal lepton flavor dependence, with $f_{1}(t)=\left(2 t^{3}+3 t^{2}-6 t^{2} \ln t-6 t+1\right) /(t-1)^{4}$ positive for any $t$, and $f_{1}(0)=1$. This manifestly positive contribution is the dominant one for $a_{\mu}$. Contributions

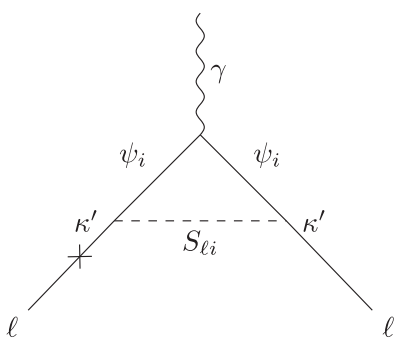

(a)

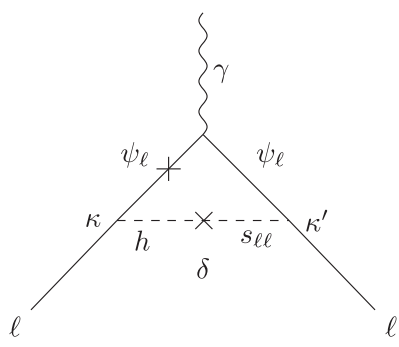

(b)
FIG. 1. Leading loop contributions to $\Delta a_{\ell}(\ell=e, \mu, \tau)$, including (a) BSM scalar fermion loops with a lepton chiral flip (cross on solid line), and (b) chirally enhanced contributions through scalar mixing (cross on dashed line), provided $\left\langle S_{\ell \ell}\right\rangle \neq 0$, and a BSM fermion $\psi_{\ell}$ chiral flip (cross on solid line).

through $Z$ and $W$ loops are parametrically suppressed as $\mathcal{O}\left(g_{2}^{2}\right)$ and by fermion mixing [31]. Comparing Eq. (5) with the muon data for a small scalar-to-fermion mass ratio $M_{S}^{2} / M_{F}^{2} \ll 1$ yields the Yukawa coupling $\alpha_{\kappa^{\prime}}$ within $(0.48 \pm 0.15)\left(\frac{M_{F}}{\mathrm{TeV}}\right)^{2}$, which is large for TeV-range fermion masses $M_{F}$. Fixing $\Delta a_{\mu}$ to the muon data (1) confirms that the corresponding contribution (5) for the electron would come out too small and with the wrong sign $\Delta a_{e} \simeq 6 \times$ $10^{-14}$ (see Fig. 2).

Additionally, chirally enhanced contributions, which are linear in the lepton mass, may arise through a portal-mediated scalar mixing where the chiral flip is shifted to a $\psi$ line [Fig. 1(b)]. The key observation is that chiral enhancement naturally explains the electron data (Fig. 2). In practice, this can be realized with either $V^{+}$or $V^{-}$. If the ground state is $V^{-}$, it must point in the electron direction (only $\left\langle S_{e e}\right\rangle \neq 0$ ), or else Eq. (1) cannot be satisfied. Overall, this leads to

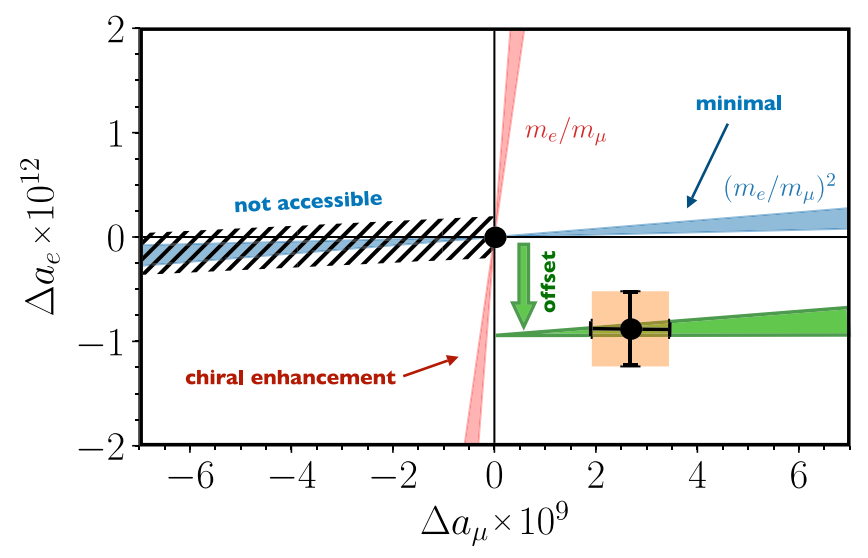

FIG. 2. Leading contributions to $\Delta a_{e, \mu}$ from Figs. 1(a) (blue band) and 1(b) (red band), which, in combination (green band), explain the electron and muon data (cross) simultaneously. The chirally enhanced offset is either flavor universal or points in the electron direction (green arrow). Band widths are indicative of a $20 \%$ mass splitting between fermion flavors from leading loops; the hatched region is inaccessible. 


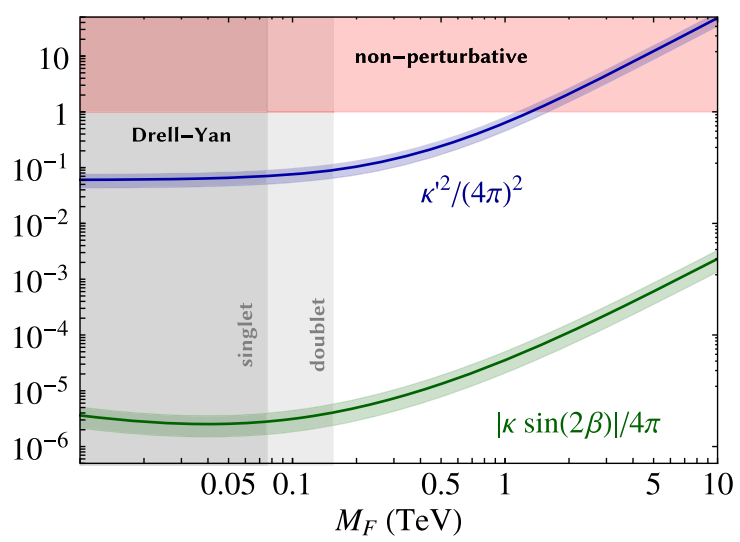

FIG. 3. Window for Yukawa and portal couplings which simultaneously explain the muon and electron data (1) as functions of the BSM fermion mass $M_{F}\left(M_{S}=0.5 \mathrm{TeV}\right.$, band width $1 \sigma$ ). Gray-shaded areas are excluded by Drell-Yan searches, while the red-shaded area indicates strong coupling. All results refer to $V^{-}$; very similar ones are found for $V^{+}$ (not shown).

$\Delta a_{e}=\frac{m_{e}}{M_{F}} \frac{\kappa \kappa^{\prime} \sin 2 \beta}{32 \pi^{2}}\left[f_{2}\left(\frac{m_{s}^{2}}{M_{F}^{2}}\right)-f_{2}\left(\frac{m_{h}^{2}}{M_{F}^{2}}\right)\right]+\frac{m_{e}^{2}}{m_{\mu}^{2}} \Delta a_{\mu}$,

where $m_{h, s}$ are the Higgs and the BSM scalar mass, and the last term accounts for Eq. (5). The loop function $f_{2}(t)=$ $\left(3 t^{2}-2 t^{2} \ln t-4 t+1\right) /(1-t)^{3}$ is positive for any $t$ and $f_{2}(0)=1$. The mixing angle $\beta$ between the scalar $s_{\ell \ell}$ and the physical Higgs $h$ is fixed via

$$
\tan 2 \beta=\frac{\delta}{\sqrt{\lambda(u+v)}} \frac{m_{h}}{m_{s}}\left(1+\mathcal{O}\left(m_{h}^{2} / m_{s}^{2}\right)\right) .
$$

In Eq. (6), the term linear in the electron mass provides a unique offset for the electron $\Delta a_{e}$, sketched in Fig. 2. It dominates parametrically over the quadratic term and can have either sign set by the Yukawas $\kappa, \kappa^{\prime}$ and the portal coupling $\delta$.

As an estimate, comparing Eq. (6) with the electron data, assuming $m_{h}^{2} / M_{F}^{2} \ll 1$ and simultaneously fixing Eq. (5) to match the muon data, we find $|\kappa \sin 2 \beta| \simeq(2.9 \pm 1.2) \times$ $10^{-4}\left(\frac{M_{F}}{\mathrm{TeV}}\right)^{2}$. The full parameter window explaining the data is indicated in Fig. 3 assuming $V^{-}$. Corrections from $Z$ and $W$ exchange, which contribute differently in the singlet and doublet models, are suppressed by small fermion mixing angles and are not sizeable enough to be seen in Fig. 3. Also shown are limits on $M_{F}$ (gray) from Drell-Yan processes $[30,32,33]$ and on perturbativity in $\alpha_{\kappa^{\prime}}$ (red). We observe $M_{F}$ within the range $(0.05-2) \mathrm{TeV}$ for $\alpha_{\kappa^{\prime}}$ within $\left(10^{-2}-1\right)$, with $\kappa \sin 2 \beta /(4 \pi)$ deeply perturbative (green) for small portal coupling $\delta$. The dual parameter space $\left(\kappa^{\prime} \ll \kappa\right)$ where Fig. 1(a) is replaced by the corresponding Higgs-fermion loops, is ruled out by $Z \rightarrow \ell \ell$ data [1], which constrain left-handed (right-handed) fermion mixing angles in the singlet (doublet) model to be of $\mathcal{O}\left(10^{-2}\right)$ or smaller.

If the vacuum is $V^{+}$, all lepton anomalous magnetic moments receive a chirally enhanced contribution from Fig. 1(b), similar to the first term in Eq. (6). The offset in Fig. 2 is then slightly tilted and points along the direction of the red band. Due to the smallness of the tilt, results and constraints are similar to those for $V^{-}$in Fig. 3 .

\section{RUNNING OF COUPLINGS UP TO THE PLANCK SCALE}

We now turn to the RG running of couplings and conditions under which models are stable and predictive up to the Planck scale. We normalize couplings to loop factors,

$$
\alpha_{x}=\frac{x^{2}}{(4 \pi)^{2}}, \quad \alpha_{z}=\frac{z}{(4 \pi)^{2}},
$$

where $x=g_{1}, g_{2}, g_{3}, y_{t}, y_{b}, y, \kappa, \kappa^{\prime}$ are any of the gauge, top, bottom, or BSM Yukawa couplings, and $z=\lambda, u, v, \delta$ are the quartic and portal couplings. Models are matched onto the SM at the scale set by the fermion mass. For the running above $M_{F}$, we retain all $12 \mathrm{RG}$ beta functions up to two-loop order in all couplings [34-37].

The left panel of Fig. 4 shows benchmark trajectories up to the Planck scale $M_{\mathrm{Pl}}$ for models starting in the vacuum $V^{-}$at the scale $M_{F}$. For some initial conditions $\left.\alpha_{\mathrm{BSM}}\right|_{M_{F}}$ at the low scale, such as those used in Fig. 4, we find that the running is stable up to the Planck scale. We also observe from Fig. 4 that the Higgs potential becomes stable (remains metastable) in the singlet (doublet) model. Higgs stability in the doublet model can be achieved for larger portal and quartic couplings. Some couplings in Fig. 4 run slowly all the way up to the Planck scale. Others show a slow or fast crossover to near-constant values due to near-zeros of beta functions [38] which arise from a competition between SM and BSM matter. In the absence of quantum gravity, the evolution of couplings ultimately terminates in an interacting UV fixed point corresponding to asymptotic safety (singlet benchmark), with asymptotic freedom prevailing in the weak and strong sectors $[17,18,23]$. In some cases, trajectories remain safe up to the Planck scale (doublet benchmark) but blow up at transPlanckian energies. For other initial conditions, we also find unsafe trajectories which terminate in sub-Planckian Landau poles (see Ref. [31] for a detailed study of initial conditions $\left.\left.\alpha_{\mathrm{BSM}}\right|_{M_{F}}\right)$.

The right panel of Fig. 4 shows the vacua of singlet and doublet models at the Planck scale in terms of the Yukawa couplings $\left.\left(\alpha_{\kappa}, \alpha_{\kappa^{\prime}}\right)\right|_{M_{F}}$ at the matching scale. Integrating the RG between $M_{F}$ and $M_{\mathrm{Pl}}$, we find wide ranges of models whose vacua at the Planck scale are either $V^{+}$(blue), or a stable $V$ with a metastable Higgs sector $\left(\alpha_{\lambda} \gtrsim-10^{-4}\right)$ such as in the SM (yellow) $[39,40]$. For other parameter ranges, we also find $V^{-}$(green), or unstable BSM potentials (gray), or Landau poles below the Planck scale (light red). 
(a)

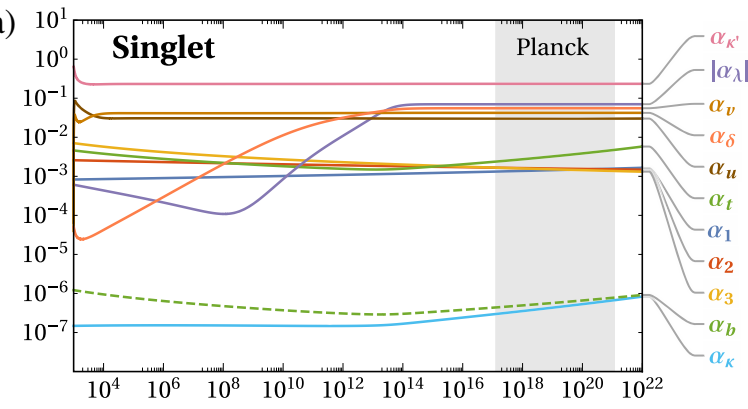

(b)

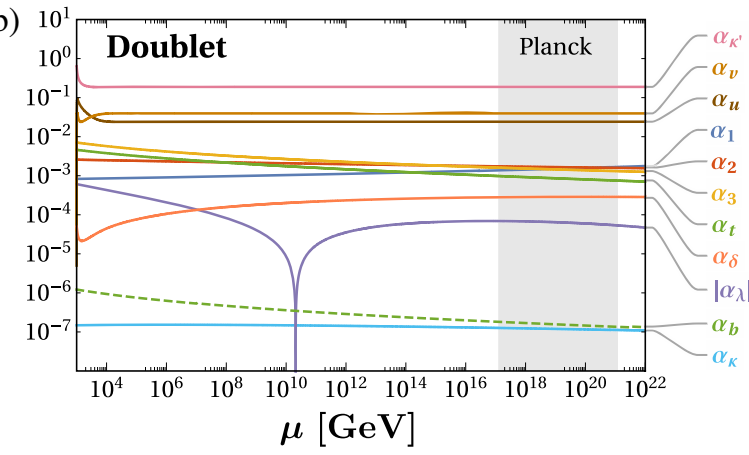

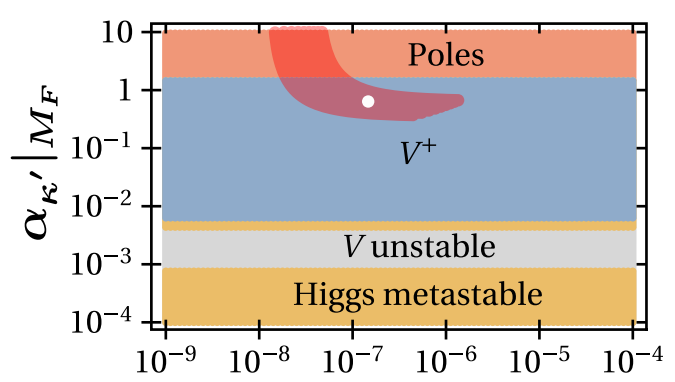

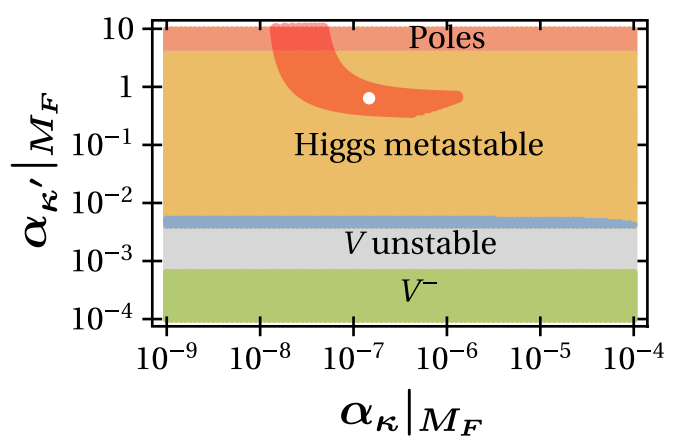

FIG. 4. Benchmark trajectories $\left(M_{F}=2 M_{S}=1 \mathrm{TeV}\right)$ between the matching scale $M_{F}$ and the Planck scale (left), and parameter scans of vacua at the Planck scale (right) for (a) the singlet model (top) and (b) the doublet model (bottom) using $\left.\left(\alpha_{\delta}, \alpha_{u}, \alpha_{v}, \alpha_{y}\right)\right|_{M_{F}}=(5,-1,4,0) \times 10^{-5}$. High-scale vacua are shown as functions of the Yukawa couplings $\left.\left(\alpha_{\kappa}, \alpha_{\kappa}^{\prime}\right)\right|_{M_{F}}$. Parameters within the red-shaded areas are compatible with Eq. (1); white dots refer to the benchmarks on the left.

Most importantly, the anomalous magnetic moments (1) are matched for couplings in the red-shaded areas which cover the $1 \sigma$ band. Constraints from Higgs signal strength [1] imply an upper bound on $\alpha_{\kappa}$, corresponding to a lower bound for the scalar mass of about $226 \mathrm{GeV}$ (for $\left.M_{F}=1 \mathrm{TeV}\right)$. Similar results are found for $V^{+}$at the low scale (not shown), except that regions with $V^{-}$in Fig. 4 turn into $V^{+}$. We conclude that models are stable and Planck-safe for a range of parameters $\left.\alpha_{\mathrm{BSM}}\right|_{M_{F}}$.

\section{COLLIDER PRODUCTION AND DECAY}

Models predict new scalars and fermions in the $\mathrm{TeV}$ energy range. Their phenomenology is characterized by an enlarged flavor sector with a large Yukawa coupling $\kappa^{\prime}$ and moderate or small couplings $\kappa, \delta$. We identify collider signatures through production and decay [31]. We denote the fermions in the singlet model by $\psi_{s}^{-1}$ and the isospin components in the doublet model by $\psi_{d}^{0}$ and $\psi_{d}^{-1}$; superscripts show electric charge. The $\psi_{d}^{0}$ is lighter than the $\psi_{d}^{-1}$ by $\Delta m=M_{\psi^{-1}}-M_{\psi^{0}}=g_{2}^{2} \sin \theta_{W}^{2} m_{Z} /(8 \pi) \simeq$ $0.4 \mathrm{GeV}$ [41]. All fermion flavors can be pair-produced in $p p$ and $\ell \ell$ machines via $s$-channel $\gamma$ or $Z$ exchange, and through $W^{ \pm}$exchange at $p p$ colliders (doublet model only). Lepton colliders allow for pair production from $t$-channel $S$ at order $\kappa^{\prime 2}$, which is sizable (see Fig. 3). Single- $\psi$ production together with a lepton arises from $s$-channel $Z$ - and $W$-boson contributions via fermion mixing. $S$ production occurs only via the Higgs portal, or at lepton colliders with $t$-channel $\psi$ in association with $h$ at order $\kappa \kappa^{\prime}$ or in pairs at order $\left(\kappa^{\prime}\right)^{2}$.

If kinematically allowed, the charged fermions decay as $\psi^{-1} \rightarrow S \ell$, and the neutral ones as $\psi_{d}^{0} \rightarrow S \nu$. If these channels are closed, the $\psi^{-1}$ decays to a Higgs plus a lepton instead. The decay rate $\Gamma\left(\psi^{-1} \rightarrow h \ell^{-}\right)=$ $\frac{\kappa^{2}}{64 \pi} M_{F}\left(1-m_{h}^{2} / M_{F}^{2}\right)^{2}$ provides the lifetime estimate $\Gamma^{-1} \sim 10^{-27}\left(1 / \alpha_{\kappa}\right)\left(1 / M_{F}[\mathrm{TeV}]\right)$ s. The neutral fermion $\psi_{d}^{0}$ cascades down slower, yet still promptly through $W$ emission with $\psi_{d, i}^{0} \rightarrow \psi_{d, i}^{-1 *} W^{+*} \rightarrow h \ell_{i}^{-} W^{+*}$. If kinematically allowed, the BSM scalars $S$ undergo tree-level decay into $\psi \bar{\psi}$ via $y$, and into $\psi \ell$ via $\kappa^{\prime}$. At one loop, the decays $S \rightarrow \gamma \gamma, Z Z, Z \gamma$, and $S \rightarrow W W$ (doublet model only) arise from $y$. Although there is no genuine lepton flavor violation (LFV), as flavor in the $S$-decay process is conserved, the mixing between the $\psi$ and the SM leptons introduces very distinct LFV-like final states $S_{i j} \rightarrow \ell_{i}^{ \pm} \ell_{j}^{\mp}$. The LFV-like decays at the order $\kappa \kappa^{\prime} v_{h} / M_{F}$ or $\left(\kappa^{\prime}\right)^{2}\left(v_{s} v_{h} / 2 M_{F}\right)^{2}$ are the leading ones for negligible $y$ and $M_{S} / M_{F} \ll 1$.

\section{DISCUSSION}

We have shown that extensions of the standard model with new vectorlike leptons and singlet matrix scalar fields (2) and (3) explain the muon and electron anomalous magnetic moments (1) simultaneously. Yukawa couplings mixing SM and BSM matter and a Higgs portal coupling are instrumental to generate both minimal (5) and chirally enhanced (6) contributions, which, when taken together, 
match the present data (Fig. 2). Also, the mechanism generating anomalous magnetic moments is rather natural and not fine-tuned to the data. In fact, our models can in principle accommodate deviations $\Delta a_{\mu}$ and $\Delta a_{e}$ in the halfplane spanned by the minimal and chirally enhanced contributions, as indicated in Fig. 2.

Further features unlike the SM are a stable Higgs potential and well-behaved running couplings up to the Planck scale. This includes asymptotically safe extensions of the SM which, for the first time, match the measured values of all gauge couplings and the Higgs, top, and bottom masses, and models which may run into poles or instabilities at transPlanckian energies. Also, some parameter settings can explain the data but are unsafe at high energies due to poles prior to the Planck scale (Fig. 4). We thus see very clearly how the high-energy behavior offers an additional selection criterion for models and their low-energy BSM parameters. Further predictions are a strongly and a weakly coupled Yukawa sector, and new matter fields with masses in the $\mathrm{TeV}$ range (Fig. 3), which can be tested at colliders.

From the viewpoint of lepton universality, it is worth noting that a manifest breaking has been instrumental in all previous models explaining both anomalies. As a proof of principle, however, our models find that any breaking beyond SM Yukawas is not mandatory. In a related vein, we also stress that lepton universality in itself is not key for asymptotic safety. In fact, it would be straightforward to explicitly break lepton flavor universality in alterations of models while maintaining predictions for both anomalies, and without spoiling a well-behaved high-energy behavior.

Another aspect which sets our models apart from any previous ones explaining both anomalies is that we also predict the deviation of the tau anomalous magnetic moment from its standard model value. This can be done solely using the data and the vacuum, and is insensitive to any other details. Specifically, provided the ground state distinguishes electron flavor, we have

$$
\Delta a_{\tau} \equiv a_{\tau}^{\text {exp }}-a_{\tau}^{\mathrm{SM}}=(7.5 \pm 2.1) \times 10^{-7},
$$

and $\Delta a_{\tau}=(8.1 \pm 2.2) \times 10^{-7}$ otherwise. Although the present limit on $\Delta a_{\tau}$ is 4 orders of magnitude away [1], it would be very interesting to test these predictions in the future. We also note that with small $C P$ phases, the electric dipole moment of the electron can be as large as the present bound, $d_{e}<1.1 \times 10^{-29} \mathrm{ecm}$ [42]. In settings with flavoruniversal vacua, the bound extends to all lepton electric dipole moments $d_{\ell}$, which would make an experimental check for the muon and the tau very challenging.

Finally, we comment on asymptotic safety as a guiding principle for model building. Vectorlike fermions alongside singlet matrix scalar fields and their Yukawa interactions are established ingredients in settings with perturbatively exact asymptotic safety, and appear prominently in templates for asymptotically safe SM extensions. Here, we have extended earlier ideas by additionally allowing for new Yukawa and portal interactions between SM and BSM matter. Curiously, these new interactions not only improve the high-energy behavior (Fig. 4) in the spirit of asymptotic safety, but also generate anomalous magnetic moments (Fig. 1) which can match the data naturally (Fig. 2). It would thus seem interesting to further explore the potential of models inspired by asymptotic safety for flavor and particle physics.

\section{ACKNOWLEDGMENTS}

This work is partly supported by the DFG Research Unit FOR 1873, "Quark Flavour Physics and Effective Field Theories." G. H. and D. L. thank the SLAC Theory Group for hospitality during the final stages of this work.

Note added.-The possibility of rendering $\Delta a_{\mu}$ insignificant has recently been suggested by a lattice determination of the hadronic vacuum polarization [43]. Note, though, that these findings are in tension with electroweak data $[44,45]$ and other lattice studies, which requires further scrutiny [46].
[1] Particle Data Group, Review of particle physics, Phys. Rev. D 98, 030001 (2018).

[2] D. Hanneke, S. Fogwell, and G. Gabrielse, New Measurement of the Electron Magnetic Moment and the Fine Structure Constant, Phys. Rev. Lett. 100, 120801 (2008).

[3] R. H. Parker, C. Yu, W. Zhong, B. Estey, and H. Mller, Measurement of the fine-structure constant as a test of the standard model, Science 360, 191 (2018).

[4] F. Jegerlehner, Muon $g-2$ theory: The hadronic part, EPJ Web Conf. 166, 00022 (2018).
[5] M. Davier, Update of the hadronic vacuum polarisation contribution to the muon $g-2$, Nucl. Part. Phys. Proc. 287-288, 70 (2017).

[6] H. Davoudiasl and W. J. Marciano, Tale of two anomalies, Phys. Rev. D 98, 075011 (2018).

[7] J. Liu, C. E. M. Wagner, and X.-P. Wang, A light complex scalar for the electron and muon anomalous magnetic moments, J. High Energy Phys. 03 (2019) 008.

[8] S. Gardner and X. Yan, Light scalars with lepton number to solve the $(g-2)_{e}$ anomaly, arXiv:1907.12571. 
[9] M. Bauer, M. Neubert, S. Renner, M. Schnubel, and A. Thamm, Axion-Like Particles, Lepton-Flavor Violation and a New Explanation of $a_{\mu}$ and $a_{e}$, Phys. Rev. Lett. 124, 211803 (2020).

[10] B. Dutta and Y. Mimura, Electron $g-2$ with flavor violation in MSSM, Phys. Lett. B 790, 563 (2019).

[11] M. Endo and W. Yin, Explaining electron and muon $g-2$ anomaly in SUSY without lepton-flavor mixings, J. High Energy Phys. 08 (2019) 122.

[12] M. Badziak and K. Sakurai, Explanation of electron and muon $g-2$ anomalies in the MSSM, J. High Energy Phys. 10 (2019) 024.

[13] A. Crivellin, M. Hoferichter, and P. Schmidt-Wellenburg, Combined explanations of $(g-2)_{\mu, e}$ and implications for a large muon EDM, Phys. Rev. D 98, 113002 (2018).

[14] A. Crivellin and M. Hoferichter, Combined explanations of $(g-2)_{\mu},(g-2)_{e}$ and implications for a large muon EDM, in Proceedings of 54th Rencontres de Moriond on Electroweak Interactions and Unified Theories (Moriond EW 2019) La Thuile, Italy, edited by E.Auge et al. (ARISF, 2019), p. 462, https://arxiv.org/pdf/1905.03789.pdf.

[15] X.-F. Han, T. Li, L. Wang, and Y. Zhang, Simple interpretations of lepton anomalies in the lepton-specific inert two-Higgs-doublet model, Phys. Rev. D 99, 095034 (2019).

[16] M. Abdullah, B. Dutta, S. Ghosh, and T. Li, $(g-2)_{\mu, e}$ and the ANITA anomalous events in a three-loop neutrino mass model, Phys. Rev. D 100, 115006 (2019).

[17] A. D. Bond, G. Hiller, K. Kowalska, and D. F. Litim, Directions for model building from asymptotic safety, J. High Energy Phys. 08 (2017) 004.

[18] K. Kowalska, A. Bond, G. Hiller, and D. Litim, Towards an asymptotically safe completion of the standard model, Proc. Sci. EPS-HEP2017 (2017) 542.

[19] D. F. Litim and F. Sannino, Asymptotic safety guaranteed, J. High Energy Phys. 12 (2014) 178.

[20] K. G. Wilson, Renormalization group and critical phenomena: 1. Renormalization group and the Kadanoff scaling picture, Phys. Rev. B 4, 3174 (1971).

[21] D. Bailin and A. Love, Asymptotic near freedom, Nucl. Phys. B75, 159 (1974).

[22] S. Weinberg, Ultraviolet divergences in quantum theories of gravitation, in General Relativity: An Einstein Centenary Survey, edited by S. W. Hawking and W. Israel (Cambridge University Press, Cambridge, England, 1979), p. 790.

[23] A. D. Bond and D. F. Litim, Theorems for asymptotic safety of gauge theories, Eur. Phys. J. C 77, 429 (2017).

[24] A. D. Bond and D. F. Litim, Price of Asymptotic Safety, Phys. Rev. Lett. 122, 211601 (2019).

[25] A. D. Bond, D. F. Litim, G. Medina Vazquez, and T. Steudtner, UV conformal window for asymptotic safety, Phys. Rev. D 97, 036019 (2018).

[26] A. D. Bond, D. F. Litim, and T. Steudtner, Asymptotic safety with Majorana fermions and new large $N$ equivalences, Phys. Rev. D 101, 045006 (2020).

[27] A. D. Bond and D.F. Litim, More asymptotic safety guaranteed, Phys. Rev. D 97, 085008 (2018).

[28] A. D. Bond and D. F. Litim, Asymptotic Safety Guaranteed in Supersymmetry, Phys. Rev. Lett. 119, 211601 (2017).
[29] D. Barducci, M. Fabbrichesi, C. M. Nieto, R. Percacci, and V. Skrinjar, In search of a UV completion of the standard model: 378,000 models that don't work, J. High Energy Phys. 11 (2018) 057.

[30] G. Hiller, C. Hormigos-Feliu, D. F. Litim, and T. Steudtner, Asymptotically safe extensions of the standard model with flavour phenomenology, in Proceedings of 54th Rencontres de Moriond on Electroweak Interactions and Unified Theories (Moriond EW 2019) La Thuile, Italy, edited by E. Auge et al. (ARISF, 2019), p. 462, https://arxiv.org/pdf/ 1905.11020.pdf.

[31] G. Hiller, C. Hormigos-Feliu, D. F. Litim, and T. Steudtner, Model building from asymptotic safety with Higgs and flavor portals, arXiv:2008.08606.

[32] D. S. M. Alves, J. Galloway, J. T. Ruderman, and J. R. Walsh, Running electroweak couplings as a probe of new physics, J. High Energy Phys. 02 (2015) 007.

[33] M. Farina, G. Panico, D. Pappadopulo, J. T. Ruderman, R. Torre, and A. Wulzer, Energy helps accuracy: Electroweak precision tests at hadron colliders, Phys. Lett. B 772, 210 (2017).

[34] M. E. Machacek and M. T. Vaughn, Two loop renormalization group equations in a general quantum field theory: 1 . Wave function renormalization, Nucl. Phys. B222, 83 (1983).

[35] M. E. Machacek and M. T. Vaughn, Two loop renormalization group equations in a general quantum field theory: 2 . Yukawa couplings, Nucl. Phys. B236, 221 (1984).

[36] M. E. Machacek and M. T. Vaughn, Two loop renormalization group equations in a general quantum field theory: 3 . Scalar quartic couplings, Nucl. Phys. B249, 70 (1985).

[37] M.-x. Luo, H.-w. Wang, and Y. Xiao, Two loop renormalization group equations in general gauge field theories, Phys. Rev. D 67, 065019 (2003).

[38] B. Holdom, Techniodor, Phys. Lett. 150B, 301 (1985).

[39] G. Degrassi, S. Di Vita, J. Elias-Miro, J. R. Espinosa, G. F. Giudice, G. Isidori, and A. Strumia, Higgs mass and vacuum stability in the standard model at NNLO, J. High Energy Phys. 08 (2012) 098.

[40] D. Buttazzo, G. Degrassi, P. P. Giardino, G. F. Giudice, F. Sala, A. Salvio, and A. Strumia, Investigating the nearcriticality of the Higgs boson, J. High Energy Phys. 12 (2013) 089.

[41] M. Cirelli, N. Fornengo, and A. Strumia, Minimal dark matter, Nucl. Phys. B753, 178 (2006).

[42] ACME Collaboration, Improved limit on the electric dipole moment of the electron, Nature (London) 562, 355 (2018).

[43] S. Borsanyi et al., Leading-order hadronic vacuum polarization contribution to the muon magnetic moment from lattice QCD, arXiv:2002.12347.

[44] A. Crivellin, M. Hoferichter, C. A. Manzari, and M. Montull, Hadronic vacuum polarization: $(g-2)_{\mu}$ versus global electroweak fits, arXiv:2003.04886.

[45] A. Keshavarzi, W. J. Marciano, M. Passera, and A. Sirlin, The muon $g-2$ and $\Delta \alpha$ connection, Phys. Rev. D 102, 033002 (2020).

[46] T. Aoyama et al., The anomalous magnetic moment of the muon in the standard model, arXiv:2006.04822. 